\title{
Un análisis de la definición y la clasificación desde los polígonos
}

\section{An analysis of definition and classification from polygons}

\author{
GUALDRÓN, Élgar ${ }^{1}$ \\ QUINTERO, Maura \\ ÁVILA-HERNANDEZ , Öscary ${ }^{3}$
}

\begin{abstract}
Resumen
Se presenta una investigación enfocada al mejoramiento del aprendizaje de la geometría y la adquisición de procesos matemáticos de pensamiento usando como pretexto la enseñanza de los polígonos. La muestra consiste de 28 estudiantes del grado quinto de una institución pública de Colombia. La metodología de investigación tiene enfoque cualitativo de tipo descriptivo, usando como técnica la observación participante. El análisis de los datos sugiere que es posible el desarrollo de procesos matemáticos de pensamiento y la mejora de aprendizajes en geometría.

Palabras clave: razonamiento geométrico, definición, clasificación, polígono
\end{abstract}

\begin{abstract}
An investigation that aims to strengthen mathematical thinking processes using the pretext of teaching polygons is presented. The sample consists of 28 fifth grade students from a public institution in Colombia. The research methodology has a descriptive qualitative approach, using participant observation as a technique. Analysis of the data suggests that the development of mathematical thinking processes using the teaching of polygons is possible.
\end{abstract}

key words: geometric reasoning, definition, classification, polygon

\section{Introducción}

La geometría es un área fundamental dentro de las matemáticas debido a su riqueza de conexiones a otras áreas de las matemáticas y su extenso uso en la vida cotidiana (Goldenberg, Cuoco \& Mark, 1998). El planteamiento de problemas en esta área, involucra situaciones diversas: la familiaridad con formas y su estructura, la ubicación, las transformaciones, y las pruebas; lo cual puede proporcionar una estructura para la comprensión de otros temas en matemáticas, en el arte, en las ciencias, la ingeniería y estudios sociales (NCTM, 2000).

En este sentido, tener conocimientos y habilidades adecuados en geometría es decisivo para que los estudiantes se preparen para la educación superior y enriquecer su vida personal y profesional en un mundo globalizado y competitivo (Russell, 2018). Desafortunadamente, muchos estudiantes experimentan dificultades para aprender conceptos de geometría (Clements, 2003; Gonzales et al., 2000; Hock, Tarmizi, Yunus \& Ayub, 2015; Wu \& Ma,

\footnotetext{
${ }^{1}$ Doctor en Didáctica de las Matemáticas. Docente de la Universidad de Pamplona (Colombia), Departamento de Matemáticas. Director del grupo de Investigación EDUMATEST_Universidad de Pamplona. egualdron@unipamplona.edu.co

${ }^{2}$ Magister en Educación. Docente de la IE Técnica Agropecuaria Vicente Hondarza (Colombia). mquintero61@unab.edu.co

${ }^{3}$ Doctorando en Educación. Facultad de Humanidades \& Educación. Universidad de Los Andes (Venezuela). arxiv.oscary@gmail.com
} 
2006). Estas dificultades incluyen la inexperiencia en los educandos para aplicar conceptos geométricos básicos apropiadamente a contextos de resolución de problemas, así como identificar un cuadrado como un tipo especial de rectángulo (Fujita, 2012; Hock et al., 2015).

El interés por el estudio del proceso de enseñanza aprendizaje de las matemáticas, y en específico de la geometría, surge al observar, a lo largo de la experiencia como docentes, las dificultades que presentan los estudiantes, en el nivel educativo de primaria, en el desarrollo de potentes razonamientos y el aprendizaje de conceptos; todo esto ligado a la falta de desarrollos plausibles de procesos matemáticos de pensamiento (Quintero, 2020). Igualmente, en el contexto de la didáctica de las matemáticas el arte de la definición desempeña un rol trascendental; junto con el acervo argumentativo e ideario aritmético que anidan en el educando (Ávila, 2020).

"La prueba Saber $3^{\circ}, 5^{\circ}$ y $9^{\circ}$ es una evaluación estandarizada, realizada anualmente por el ICFES (Instituto Colombiano para la Evaluación de la Educación), con el fin de evaluar la educación básica primaria y básica secundaria, y brindar información para el mejoramiento de la calidad de la educación" (ICFES,2011, p. 2).

Al cotejar lo manifestado por los docentes con los resultados de la Prueba Saber $5^{\circ}$ en los años 2016 y 2017 (ICFES, 2017b), se puede notar que existe concordancia; es decir, las dificultades manifiestas por los docentes se ven reflejadas en los resultados en matemáticas de dicha prueba, particularmente, en las diferentes competencias evaluadas. En dicha prueba, " los niveles de desempeño consisten en una descripción cualitativa sobre lo que el estudiante es capaz de hacer cuando se enfrenta a preguntas de distintos rangos de dificultad, en una situación de contexto específica" (ICFES, 2017a, p. 4-5). "Las descripciones explican lo que saben y saben hacer los estudiantes en cada nivel de desempeño: insuficiente (no supera las preguntas de menor complejidad de la prueba), mínimo (muestra un desempeño mínimo en las competencias exigibles para el área y grado evaluados), satisfactorio (muestra un desempeño adecuado en las competencias exigibles para el área y grado evaluados) y avanzado (muestra un desempeño sobresaliente en las competencias esperadas para el área y grado evaluados)" (ICFES, 2017a, p. 5).

A nivel nacional, en el año 2016, el 65\% de los estudiantes de quinto grado se ubicó en los niveles de desempeño insuficiente y mínimo; mientras en el año 2017, los resultados fueron más desalentadores, hubo un $72 \%$ de los estudiantes ubicados en dichos niveles.

A nivel rural, en matemáticas, los resultados en las pruebas son más desalentadores, por ejemplo, en la zona del sur del departamento de Bolivar, los estudiantes de $5^{\circ}$ grado, en los años 2016 y 2017, se ubicaron en promedio en el $85 \%$ en los niveles más bajos: insuficiente y mínimo. En particular, en geometría, los resultados son similares; los conceptos geométricos y el uso de procesos matemáticos de pensamiento como herramienta en la resolución de problemas es una de las mayores dificultades.

Una probable causa de lo mencionado anteriormente es la transmisión de contenidos en geometría, por parte de los docentes, sin profundizar, ni estimular el razonamiento a través de la exploración y un descubrimiento guiado (Gamboa \& Ballestero, 2010). De acuerdo con lo anterior, la incomprensión de los conceptos produce dificultades en el desempeño académico, pues si no se utilizan los métodos y estrategias adecuadas, los estudiantes no podrán dar definiciones ni clasificaciones de objetos geométricos de una manera amplia y clara, es decir sus argumentos serán incompletos sin tener sentido alguno (Gualdrón, 2011); por lo tanto, el estudiante no tiene la habilidad de conectar los temas vistos en la escuela con la realidad de su vida cotidiana. En este sentido, uno de dichos temas es el polígono (en este trabajo incluimos también el triángulo y el cuadrilátero) el cual, si no se enseña de manera adecuada, dificulta el desarrollo de otros conceptos geométricos que hacen parte del currículo escolar del educando. 
Entre otras cosas, bajo este panorama académico, surge la necesidad de dar respuesta, por lo menos en parte, a las dificultades presentadas por los estudiantes de quinto grado de una Institución Educativa Técnica de la región del sur del departamento de Bolivar y que se plasma mediante la pregunta problematizadora ¿Es posible fortalecer los procesos matemáticos de pensamiento: definición y clasificación en estudiantes de $5^{\circ}$ grado usando como pretexto la enseñanza de los conceptos de polígono, de triángulo y de cuadrilátero?

En esta investigación se proyecta el fortalecimiento de los procesos matemáticos de pensamiento: definición y clasificación, teniendo como objeto de estudio el polígono, el triángulo y el cuadrilátero, los cuales son parte fundamental de la geometría. Se planeó, con el fin de alcanzar dicho fortalecimiento, una intervención pedagógica mediante una unidad didáctica diseñada bajo los preceptos de los modelos de Van Hiele (1957, 1986, 1999), de Vinner \& Hershkowitz (1983), y los preceptos de Gutiérrez \& Jaime (1998). La intervención se realizó con los estudiantes de quinto grado de la mensionada institución quienes componen la muestra seleccionada.

El trabajo de investigación desarrollado permite contribuir a la tarea de clarificar las maneras de llevar a cabo el fortalecimiento de la definición y la clasificación como procesos matemáticos de pensamiento; además, permite contribuir al proceso de enseñanza y de aprendizaje de conceptos geométricos.

\subsection{Marco teórico}

El propósito de este apartado es dar a conocer, de manera concisa, los referentes teóricos que soportan la investigación y que además permitieron la obtención de resultados.

\subsubsection{El modelo de Van Hiele}

La estructura de este modelo se fundamenta bajo los principios de que: en el proceso de aprendizaje de la geometría, el razonamiento de los estudiantes evoluciona gradualmente mediante unos niveles secuenciados y ordenados sin que se salte ninguno (Van Hiele, 1986).

El modelo de Van Hiele lo constituyen dos elementos fundamentales: los niveles de razonamiento y las fases de aprendizaje. Los niveles son la manifestación de los razonamientos geométricos de los estudiantes desde que se inicia el aprendizaje en un concepto específico hasta que logran su máximo desarrollo intelectual. Las fases de aprendizaje son los momentos que se sugieren para diseñar y gestionar las clases, de tal forma que los estudiantes puedan avanzar en sus razonamientos.

\section{Los niveles de razonamiento}

La formulación de los niveles han pasado por un proceso de evolución desde que fueron planteados (Pegg \& Davey, 2007) puesto que las ideas del propio Van Hiele evolucionaron, desde su propia experiencia y la de otros investigadores y los resultados obtenidos acerca de los niveles. Dicho lo anterior, entre otras cosas, se puede decir que los niveles en un principio se enumeraron desde el nivel 0 al $4 \mathrm{y}$, posteriormente, una totalmente diferente, desde el nivel 1 al 5; de acuerdo con lo consultado, se trata solamente de una cuestión de etiqueta, es decir, las características de los niveles no cambiaron. Con estas indicaciones, y para mayor comodidad, se ha decidido tomar los niveles de 1 a 5, al mismo tiempo que se ha prescindido para este estudio de los tres últimos niveles, puesto que los estudiantes de la Educación Básica, en la mayoría de los casos, sólo alcanzan los niveles de razonamiento1 y 2 (Jaime, 1993; Gualdrón 2011); esto no quiere decir que algunos estudiantes no puedan alcanzar el nivel 3 o estar transitando del nivel 2 al 3, es decir no cumplir todas las características que lo definen.

En este sentido, se presenta las características (descriptores) de los tres primeros niveles:

Nivel 1: El estudiante distingue, de manera global, las figuras geométricas por su forma, ni diferencia sus partes ni componentes. Por el contrario, es capaz de reproducir una figura dada o incluso reconocerla por su apariencia física. No puede explicar las propiedades matemáticas de las figuras, pero si puede realizar descripciones 
figurales usando argumentos de tipo visual y usando comparaciones con elementos que le son familiares de su entorno. El lenguaje geométrico elemental es muy exiguo para comunicar ideas en torno a las figuras geométricas.

Nivel 2: El estudiante distingue y analiza los elementos y propiedades matemáticas de las figuras geométricas, pero no logra establecer clasificaciones de figuras dentro de la misma familia. Por el contrario, puede declarar propiedades de las figuras usando la experimentación. Comprende los elementos constitutivos de una definición en geometría, pero, en algunos casos, no le es posible plantearlas.

Nivel 3: El estudiante identifica las figuras por sus propiedades matemáticas y distingue cómo se relacionan unas con otras. Comprende las condiciones suficientes y necesarias que deben cumplir las definiciones de las figuras geométricas. Puede seguir demostraciones de propiedades matemáticas pero se le dificulta entenderlas integralmente. Se le dificulta realizar razonamientos lógicos formales por lo que no comprende el sistema axiomático en matemáticas. Le es posible comprender el cómo unas propiedades se deducen de otras.

\section{Las fases de aprendizaje}

Las fases se asocian a cada nivel de razonamiento y el diseño de la enseñanza se realiza teniendo en cuenta dichas fases. Se pueden alcanzar niveles más altos si se consiguen las experiencias didácticas correctas (Van Hiele, 1986).

A continuación, se dará a conocer, de manera sucinta, las fases de aprendizaje de Van Hiele (Van Hiele, 1986), pero bajo estudios actuales de Gualdrón (2011) quien ha matizado las mismas y son pertinentes para el actual estudio:

\section{“Fase 1: Información}

El profesor en esta fase dialoga con los estudiantes y les informa del tema que van a desarrollar, los objetivos de estudio y las actividades que planea desarrollar. También, el profesor tendrá la oportunidad de enterarse de los conocimientos previos que tienen los estudiantes que son pertinentes para el desarrollo del nuevo, además los estudiantes conocerán la futura dirección a la cual el nuevo tema los conducirá.

\section{Fase 2: Orientación dirigida}

En esta fase el profesor presenta gradualmente el material, compuesto de tareas cortas que generen respuestas específicas, que ha preparado cuidadosamente para que los estudiantes exploren el nuevo tema de estudio. Dicha exploración incluye que los estudiantes descubran y aprendan las posibles relaciones o componentes básicos que deben formar.

\section{Fase 3: Explicitación}

Es en esta fase donde se realiza un afianzamiento del tópico que se está estudiando, el cual incluye el manejo adecuado del lenguaje técnico, características, propiedades, relaciones que se han observado y analizado. El profesor debe propiciar la discusión entre él y los estudiantes y entre los mismos estudiantes. Esta fase no debe entenderse como una más cronológicamente hablando, sino más bien una fase que complementa a las otras.

\section{Fase 4: Orientación libre}

Es una fase en la cual el profesor debe preparar tareas que sean novedosas, diferentes a las que ha propuesto antes (con muchos pasos e incluso más complejas), que tengan diferentes vías de resolución, que le permitan a los estudiantes establecer relaciones entre los objetos que están estudiando. La intervención 
del profesor en esta fase debe ser mínima, de tal forma que los estudiantes intenten por sí solos buscar la solución.

\section{Fase 5: Integración}

En esta fase los estudiantes, con ayuda del profesor, realizan un resumen de todo lo aprendido, lo que les permitirá tener una visión global de los objetos y relaciones en relación al tema de estudio. Es una fase en la cual no se realiza el desarrollo de temas nuevos, sólo la recopilación y organización de los ya adquiridos" (p. 42-43).

\section{Las propiedades del Modelo de Van Hiele}

Estas propiedades son descritas en Van Hiele (1986), a continuación se dan a conocer.

Recursividad: cuando un estudiante asciende de nivel, los razonamientos en el nivel anterior no se pierden, evolucionan.

Jerarquía y secuencia de los niveles: cuando un estudiante adquiere un nuevo nivel, se supone que ya había adquirido el anterior o anteriores. La adquisición de los niveles se realiza de forma organizada.

Relación entre el lenguaje y los niveles de razonamiento: los niveles incluyen el manejo por parte de los estudiantes de un lenguaje apropiado, es decir, en la medida que avanza en el logro de niveles su lenguaje evoluciona, se hace más matemático y menos coloquial. Esto sugiere el por qué algunos estudiantes no comprenden lo que el profesor explica; justamente porque usa un lenguaje que el estudiante no ha adquirido.

Localidad de los niveles de razonamiento: cuando los estudiantes han adquirido un determinado nivel de razonamiento en un objeto geométrico específico y en otros, uno diferente. Es decir, por ejemplo, un estudiante puede estar en nivel 2 en polígonos y estar en nivel 1 en congruencia de triángulos.

Continuidad de los niveles: a pesar de que para Van Hiele el paso de un nivel a otro se manifestaba de un momento para otro, la propia evolución de sus ideas manifiestan que no sucede así, es decir, es un proceso lento. Un estudiante puede haber adquirido completamente un nivel e ir transitando lentamente hacia el siguiente.

La importancia de la instrucción en el modelo: el alcance por parte de los estudiantes de cada nivel va a depender de un óptimo diseño y gestión de la clase por parte del profesor.

Dicho lo anterior, se puede concluir que el rol de expositor del profesor debe cambiar y adquirir un papel de coordinador de las actividades propicias para alcanzar los objetivos educativos trazados; actividades necesarias para proporcionarle al estudiante un contexto adecuado para el desarrollo de su razonamiento y su paso por los diversos niveles. El rol del estudiante se ve transformado, de un receptor pasivo a un investigador activo de la información. Estos cambios de roles involucran la necesidad de que el docente manipule y reconozca el material para trabajarlo sin obstáculos, apoyando al estudiante en la exploración y elaboración de su propio conocimiento (Cabanne \& Ribaya, 2009).

\subsubsection{Procesos matemáticos de pensamiento: definición y clasificación}

En la sección anterior se describió el modelo de Van Hiele, entre otras cosas, los descriptores de nivel. Según Gutiérrez \& Jaime (1998) dichos descriptores conducen tácitamente a la idea de que cada nivel de razonamiento debe concebirse como la suma de un conjunto de procesos. Además agregan que el logro por los estudiantes de un determinado nivel de razonamiento se debe considerar sólo hasta cuando los estudiantes muestren dominio en los procesos que constituyen tal nivel. En este sentido, es legítimo pensar que para el diseño de una unidad didáctica se deberían tener en cuenta tales procesos que componen cada nivel de razonamiento. 
Los procesos matemáticos de pensamiento son para Gutiérrez \& Jaime (1998) de elevada importancia en el proceso de enseñanza y aprendizaje de la geometría; sus estudios a este respecto parten de las ideas de Hoffer (1981) y De Villiers (1987), las cuales son analizadas y asumidas, junto con otros, para la constitución de un referente en cuanto a este respecto, es decir, un listado de procesos matemáticos de pensamiento involucrados en los niveles de Van Hiele. La propuesta es la siguiente:

“1. Reconocimiento de tipos y familias de figuras geométricas, identificación de componentes y propiedades de las figuras.

2. Definición de un concepto geométrico. Este proceso puede verse en dos formas: a medida que los estudiantes formulan definiciones del concepto que están aprendiendo, y cuando los estudiantes usan una definición dada leída en un libro de texto, o escuchada del profesor u otro alumno.

3. Clasificación de figuras geométricas o conceptos en diferentes familias o clases

4. Demostración de propiedades o declaraciones, es decir, para explicar de manera convincente la forma en que dicha propiedad o declaración es verdadera" (Gutiérrez \& Jaime, 1998, p. 29).

En lo mencionado, "cada proceso es un componente de dos o más niveles de razonamiento" (Gutiérrez \& Jaime, 1998, p. 29); y "la manera como un estudiante considera y usa los procesos es un indicador del nivel de su razonamiento" (Gutiérrez \& Jaime, 1998, p. 29-30).

En lo que sigue, se hace referencia a las características que cumplen los procesos en cada nivel de razonamiento, particularmente en los niveles 1 y 2, tomadas de Gutiérrez \& Jaime (1998):

1. El reconocimiento de los estudiantes en el nivel 1 se limita a los atributos físicos y globales de figuras. A veces usan vocabulario geométrico (no tan común en la básica primaria), pero dichos términos tienen un significado visual más que uno matemático. Por ejemplo, al describir un rectángulo, algunos estudiantes usan el término "alargado", para así diferenciarlo del cuadrado. En otros casos, son capaces de notar correctamente algunas propiedades matemáticas de las figuras, pero estas son propiedades simples, como el número de lados. Sin embargo, los estudiantes de nivel 2 o superior pueden usar y reconocer propiedades matemáticas de conceptos geométricos. Es importante notar que la habilidad de reconocimiento no discrimina entre estudiantes en los niveles de Van Hiele 2, 3 o 4.

2. Los estudiantes en el nivel 1 no pueden usar definiciones matemáticas dadas. Las definiciones solo las pueden formular y consisten en descripciones de atributos físicos de la figura que están observando, como "redondo" o "más largo que más ancho" y quizás alguna propiedad matemática básica. Cuando los estudiantes en el nivel 2 reciben una definición matemática, y conocen todas las propiedades contenidas en la definición, pueden usarla. Estos estudiantes, sin embargo, pueden experimentar dificultades al usar algunas expresiones lógicas, como " $\mathrm{y}$ ", "o" o "al menos". Los estudiantes en el nivel 2 no entienden el estructura lógica de las definiciones (es decir, conjuntos de propiedades necesarias y suficientes del concepto definido), entonces cuando se les pide una definición que no ha sido aprendida de memoria, a menudo proporcionan una larga lista de propiedades del concepto, sin darse cuenta de redundancias.

3. Los estudiantes en el nivel 1 pueden entender clasificaciones exclusivas, ya que no aceptan ni reconocen ningún tipo de relación lógica entre clases ni, muchas veces, entre dos elementos de la misma clase que tienen bastante diferencia en la apariencia física. En el presente estudio se acepta la posición de Gutiérrez \& Jaime (1998), pero dada la circunstancia particular de los estudiantes de la muestra, que nunca han visto de manera detallada y amplia a los polígonos, a los triángulos y a los cuadriláteros, se decidió trabajar sólo clasificaciones inclusivas, las que tuvimos en cuenta también en el desarrollo del nivel 2 de razonamiento. 
4. Los estudiantes en el nivel 1 no pueden entender el concepto de demostración. Para estudiantes en nivel 2, una demostración típica consiste en una verificación experimental de la verdad de la propiedad en uno o pocos casos. Dependiendo del grado de adquisición de los estudiantes de esta habilidad, pueden estar convencidos con solo un ejemplo especial, o pueden necesitar un conjunto de ejemplos más elaborado. Por otra parte, para este estudio, se considera a la demostración, en el nivel 2, como la argumentación mediante propiedades matemáticas que el estudiante hace para justificar una respuesta.

La tabla $\mathrm{N}^{\circ} 1$ resume las características de los procesos matemáticos de pensamiento para los niveles 1 y 2 de Van Hiele que se tendrán en cuenta en el presente estudio y que tuvieron como fundamento a Gutiérrez \& Jaime (1998).

Tabla 1

Características de los procesos matemáticos de pensamiento de los niveles 1 y 2 de Van Hiele.

\begin{tabular}{lll}
\hline Procesos & Nivel 1 & Nivel $\mathbf{2}$ \\
\hline $\begin{array}{l}\text { Reconocimiento y } \\
\text { Descripción }\end{array}$ & $\begin{array}{l}\text { Atributos físicos (posición, } \\
\text { forma, tamaño) }\end{array}$ & $\begin{array}{l}\text { Propiedades } \\
\text { matemáticas }\end{array}$ \\
\hline Uso de definiciones & ------------ & Definiciones con \\
& & estructuras simples \\
\hline Formulación de & Listado de propiedades & Listado de propiedades \\
Definiciones & físicas & matemáticas \\
\hline Clasificación & Inclusiva basada en & Inclusiva basada en \\
& atributos físicos & atributos matemáticos \\
\hline Demostración & ------------- & Verificación con \\
& & ejemplos \\
\hline
\end{tabular}

Fuente: elaboración propia por adaptación de Gutiérrez \& Jaime (1998)

En conclusión, como ya se dijo, en el diseño de la unidad didáctica se usó, además de los descriptores de nivel de razonamiento y las fases de aprendizaje, las características de los procesos matemáticos de pensamiento en cada uno de los niveles 1 y 2 de razonamiento; además del modelo de Vinner \& Hershkowitz. También, recordar que este estudio sólo se enfocó en los procesos definición (uso y formulación) y clasificación.

\subsubsection{El Modelo de Vinner \& Hershkowitz: formación de conceptos geométricos}

Con frecuencia, tanto profesores como libros de texto exponen el concepto de polígono, triángulo y cuadrilátero mediante la definición matemática, el planteamiento de ejemplos gráficos que los representan, y terminan proponiendo unos cuantos dibujos de figuras para que los estudiantes decidan el tipo de polígono. En algunos casos, se plantean ejercicios de memorización de la definición. Sea en el caso de los profesores o en libros de texto, se suele manifestar el énfasis en las definiciones, más que en los mismos ejemplos. Esta tendencia va en contravía de lo que sería deseable; de acuerdo a Vinner $(1983,1991)$, los ejemplos impactan en mayor medida los aprendizajes ya que tienen un mayor efecto en la construcción del concepto.

Según el mismo Vinner, cuando un estudiante escucha o lee el nombre de un concepto, al que ya ha tenido acercamiento, se evoca de manera casi inmediata, más que la definición, la representación gráfica o la imagen mental del mismo. Es en este punto donde Vinner adopta el nombre de imagen del concepto. En el caso de los polígonos, triángulos y cuadriláteros la imagen del concepto es la que crean mentalmente los estudiantes y está formada por diversas imágenes, dibujos o representaciones para ser recordadas como ejemplos de dichos conceptos.

En términos de Vinner, la imagen de un concepto es adecuada si le permite al estudiante discernir sin equivocaciones todos los ejemplos de ese concepto. En el caso concreto del presente estudio, por ejemplo, la 
imagen del concepto de triángulo, principalmente en la educación básica primaria, está conformada por una pequeña variedad triángulos, con lados casi de igual medida, y ubicados en posición estándar (uno de sus lados visto de forma horizontal a la hoja, a la que generalmente le llaman base).

De otra parte, siguiendo con la forma como se enseña el concepto de polígono, la definición del mismo es aprendida por los estudiantes a manera de letanía, donde poco o nada comprende de la misma; la situación se agudiza cuando, a pesar de saber memorísticamente la definición, no la saben usar en situaciones matemáticas concretas. En este sentido, Vinner \& Hershkowitz (1983) sugieren el nombre de definición del concepto a esta circunstancia, es decir a la definición que memorizaron los estudiantes que, en la mayoría de casos, está desligada de la imagen del concepto, lo que les impide abordar efectivamente las tareas matemáticas que se les pueda plantear. Por ejemplo, cuando se les pide la definición de cuadrado, algunos reproducen la que memorizaron diciendo "es un polígono de cuatro lados iguales y cuatros ángulos rectos"; en este grupo de estudiantes están los que logran usarla correctamente identificando cuadrados de una batería de ejemplos dada, pero también hay otro grupo que no, es el caso de los que no eligen a los cuadrados que están inclinados (por ejemplo 45 grados) por que los consideran rombos. La discrepancia expuesta pone de manifiesto le desvinculación que en muchos casos se presenta entre la imagen y la definición del concepto y, como consecuencia, el mal uso que se le pueda dar en ambos casos en el desarrollo de las tareas matemáticas formuladas por el profesor o el mismo libro de texto.

La experiencia personal y los ejemplos a los que ha tenido acceso un estudiante, tanto en la escuela como fuera de ella, marcan la calidad de la imagen de un concepto. En muchos casos, sobre todo los ejemplos, son escasos y presentados de tal forma que se puedan generar estereotipos, es decir una imagen correcta del concepto puede que no encaje en el estereotipo formado (Hershkowitz, 1990). Por ejemplo, cuando las únicas imágenes que se presentan de rectángulo son aquellas que se muestran con un par de lados más alargados que el otro par y siempre en posición estándar (sobre todo el lado más largo dibujado en la horizontal). Esto sugiere que en la enseñanza de un concepto, y particularmente su imagen, se presenten la mayor cantidad de ejemplos, tratando de evitar la generación de estereotipos, que permitan una imagen adecuada y completa.

En conclusión, las ideas sugeridas por Vinner \& Hershkowitz (1983), por Vinner $(1983,1991)$ y por Hershkowitz (1990), han permitido clarificar la manera cómo se consolida la construcción de un concepto (imagen y definición), particularmente en geometría, en el contexto escolar. En el estudio que se presenta, se tuvo en cuenta en el diseño de la unidad didáctica, con la apuesta a formar imágenes conceptuales sólidas (de polígono, triángulo y cuadrilátero). Estas ideas van en armonía con lo que plantea el modelo de Van Hiele, principalmente, no únicamente, en el primer nivel de razonamiento (visualización), donde se construye, mediante ejemplos y no ejemplos adecuados, la imagen del objeto geométrico de estudio.

\section{Metodología}

En este capítulo se presenta el enfoque metodológico que se tendrá en cuenta para realizar la investigación, los instrumentos y técnicas que se establecen para la recolección e interpretación de la información pertinente para dar respuesta a la pregunta de investigación y a los objetivos planteados.

\subsection{Tipo de investigación}

El proceso de investigación se desarrolla incorporando el enfoque propio de la investigación cualitativa, puesto que es propia del contexto educativo. Esta permite observar, explorar, analizar y describir un fenómeno en específico a partir de la interacción directa y de las diferentes situaciones que rodean al aula de clases, teniendo en cuenta los individuos estudiados y el rol del maestro, proporcionando una gran cantidad de información valiosa, con el fin de dar solución a las dificultades encontradas en el contexto. 


\subsection{Población y muestra}

El proceso metodológico incluye identificar la población y la muestra que se desea examinar, para así poder diseñar las estrategias y las actividades que permitan los progresos en los aprendizajes de los estudiantes en el contexto del aula.

\subsubsection{Población}

Arias (2006) afirma que una población es "como el conjunto finito o infinito de elementos con características comunes para los cuales serán extensivas las conclusiones de la investigación" (p. 34). La población seleccionada para esta investigación es una institución educativa técnica agrícola del sur del departamento de Bolivar, de carácter público, en el segundo semestre académico del año 2019.

\subsubsection{Muestra}

Hurtado (2000) considera la muestra como "una porción de la población que se toma para realizar el estudio, la cual se considera representativa de la población" (p. 17). En el presente estudio, la muestra seleccionada es el grado $5 \mathrm{~A}$ de dicha institución. El curso seleccionado está constituido por 28 estudiantes, que oscilan entre 10 y 12 años de edad, 18 mujeres y 10 hombres. La elección de la muestra fue estratégica (por conveniencia) dado que el profesor titular de 5A fue quien más se interesó por la investigación y permitió realizar las intervenciones planeadas.

\subsection{Técnicas e instrumentos para la recolección de la información}

Las técnicas e instrumentos que se tuvieron en cuenta en esta investigación son el diagnóstico, la observación participante, los diarios de campo y la unidad didáctica, los cuales permitieron profundizar la interpretación y los análisis de la situación problemática presentada en el contexto y así lograr los objetivos propuestos y resultados esperados para la investigación.

\subsubsection{Diagnóstico}

A pesar que el actual estudio se centra en los procesos matemáticos de pensamiento, usando los polígonos como vehículo para su desarrollo, se hace necesario indagar el estado en el que se encuentran los aprendizajes sobre polígono (presaberes) en los estudiantes de $5^{\circ} \mathrm{A}$. En este sentido, se diseñó una prueba de nueve preguntas relacionadas con los presaberes del concepto de polígono.

\subsubsection{Observación participante}

Se asume los lineamientos de Marín (2018): "el observador o investigador asume el papel de miembro del grupo, comunidad o institución que investiga y, como tal, participa de su funcionamiento cotidiano" (p. 221). Esta asunción concuerda con el paradigma cualitativo elegido, donde un miembro del equipo investigador hace parte del proceso a través de la interacción con los estudiantes, permitiendo tener un conocimiento claro y preciso de las características que expresan lo observado en cada momento de la investigación, tomando lo más relevante para cumplir con el objetivo propuesto. En concreto, para este estudio se ha usado la expresión "notas del investigador", que le permiten complementar el proceso de análisis, principalmente, del desarrollo de la unidad didáctica.

\subsubsection{Diario de campo}

Porlán \& Martín (1991) definen el diario de campo como un "instrumento de análisis del pensamiento reflexivo de profesores tanto en formación como en ejercicio" (p. 21). Se entiende al diario de campo como la descripción detallada y objetiva la información significativa de las actividades realizadas por los estudiantes después de cada una de las sesiones. 


\subsubsection{Unidad didáctica}

Según Arias (2006), los instrumentos son "los medios materiales que se emplean para recoger y almacenar la información" (p. 18). Para la recolección de la información relevante se ha diseñado una unidad didáctica teniendo en cuenta los modelos teóricos de Van Hiele (niveles 1 y 2) y de Vinner \& Hershkowitz, y los procesos matemáticos de pensamiento.

Durante este apartado se hace énfasis específicamente en qué consiste la estructura de las actividades que conforman la unidad didáctica, teniendo en cuenta los niveles de razonamiento del 1 al 2 de Van Hiele y las fases de aprendizaje respectivas como organizadoras de la enseñanza que se encuentran en el referente teórico planteado, sin dejar de lado que esta unidad es el instrumento indispensable para la recolección de la información. Por otra parte, la unidad didáctica también tuvo en cuenta la estructura del plan de área para los estudiantes de $5^{\circ}$ grado de la población objeto de estudio.

La unidad didáctica está formada por una primera actividad (denominada actividad "0") que contiene las nociones preliminares al concepto de polígono, donde solo se tiene en cuenta el nivel 1 , dado que son nociones que se abordaron en otros grados y únicamente se abordan en la fase de información, por que como ya se dijo en el marco teórico, entre otras cosas en esta fase se revisan y se aclaran dichas nociones. Luego se diseñan tres actividades denominadas construcción y uso de la definición de polígono (denominada actividad "1"), triángulos (denominada actividad " 2 "), y cuadriláteros (denominada actividad " 3 "), donde cada una de estas contiene tareas para desarrollar los niveles 1 y 2 . Cada uno de estos niveles contiene tres tareas en la fase de orientación dirigida, dos tareas en la fase de orientación libre y dos tareas en la fase de integración. Es importante recordar, como se dijo en el marco teórico, que para este estudio solo se tendrán en cuenta estas fases.

En general, un aspecto importante tenido en cuenta en el diseño de las actividades es el hecho de que la representación gráfica de los polígonos, en las diferentes actividades, se presenta en posiciones estándar y no estándar (Vinner \& Hershkowitz, 1983), con el fin de no generar estereotipos en el aprendizaje de los estudiantes.

\section{Resultados}

Una vez recolectada la información en cada una de las etapas del trabajo de investigación, se realiza el proceso de triangulación que permite profundizar en el análisis y la comprensión de los datos obtenidos, teniendo en cuenta las categorías de análisis. Al mismo tiempo, se verifican las fuentes de información como son: las observaciones realizadas (diario de campo), el nivel de desempeño alcanzado por los estudiantes durante la prueba diagnóstica, y el avance durante el desarrollo de las diferentes actividades que conforman la unidad didáctica.

\subsection{Análisis de la prueba dignóstica}

Una vez implementada la prueba dignóstica, se procedió a realizar su análisis teniendo en cuenta únicamente los razonamientos usados y si las respuestas eran correctas o no. A continuación las conclusiones:

- Los estudiantes solo reconocen polígonos que son típicamente desarrollados en los diferentes grados de la básica primaria: triángulos, cuadrados y rectángulos. En otras palabras, no reconocen como polígonos a aquellos diferentes a los triángulos, a los cuadrados y a los rectángulos. Estos resultados sugieren que la imagen conceptual de polígono es escasamente desarrollada por los profesores de grados anteriores o que los instrumentos, como el libro de texto, no lo privilegia.

- Los estudiantes sólo reconocen los triángulos, cuadrados y rectángulos en posición estándar (uno de los lados dibujado de manera horizontal a la hoja de trabajo). 
- Los estudiantes no reconocen aspectos matemáticos de los polígonos, de los triángulos, ni de los cuadriláteros. Es decir, aspectos como los ángulos, los lados, los vértices, el paralelismo y perpendicularidad de los lados.

- Los estudiantes no logran formular la definición de polígono mediante ejemplos y no ejemplos del mismo. Es decir, no identifican ni físicamente ni matemáticamente las características que lo definen. Estos resultados sugieren que, a pesar que en grados anteriores se les ha enseñado la definición del concepto, no la recuerdan o no la saben.

- Los estudiantes no reconocen clasificaciones inclusivas entre diferentes polígonos.

\subsection{Análisis de la unidad didáctica}

El análisis de las producciones de los estudiantes, una vez implementadas las actividades de la unidad didáctica, se realizó usando como categorías de análisis las que se obtuvieron usando los descriptores de nivel de Van Hiele (1957, 1986), de Burger \& Shaughnessy (1986), los procesos matemáticos de pensamiento de Gutiérrez \& Jaime (1998), y las temáticas propias del contexto, es decir el polígono, el triángulo y el cuadrilátero. Del nivel 1 de Van Hiele resultaron 10 categorías y del nivel 2 resultaron 13; fueron etiquetadas usando los numerales del 1.1 al 1.10 y del 2.1 al 2.13. En dichos numerales, el primer número indica el nivel de Van Hiele y el segundo la secuencia.

A continuación se presentan algunos ejemplos representativos de respuestas de los estudiantes en las tareas planteadas en las diferentes actividades de la unidad didáctica. Los nombres de estudiantes mensionados en el análisis son ficticios, es decir, no corresponden a los reales.

\subsubsection{En la Actividad N¹}

La tarea $\mathrm{N}^{\circ} 1$, presenta 18 ejemplos y 18 no ejemplos de polígono, tiene como objetivo de que los estudiantes formulen la definición del concepto. Se presenta la producción de Isabela quien, luego de observar y analizar los ejemplos y no ejemplos dados, logra definir acertadamente al polígono. Este tipo de producción se corresponde con la categoría 1.8 [Formulan definiciones realizando un listado de propiedades físicas].

Figura 1

Producción de Isabela en la tarea $n^{\circ} 1$ de la actividad 1

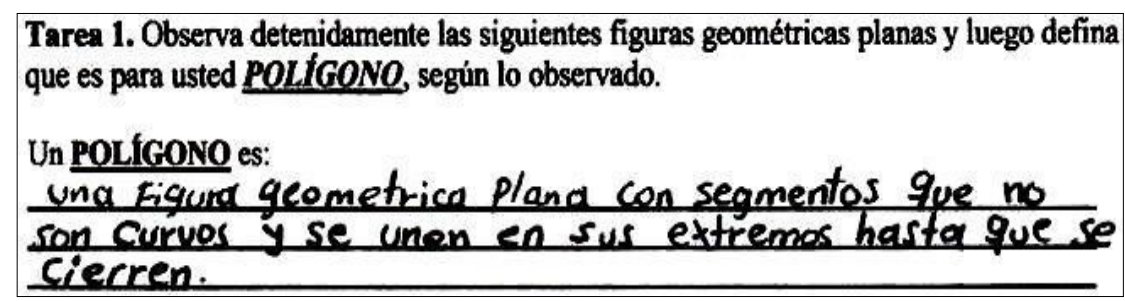

Fuente: elaboración propia

La tarea $\mathrm{N}^{\circ} 8$ tiene como finalidad la presentación de las definiciones del trapecio, cuadrado, rombo, triángulo y rectángulo; así como también, su uso en la determinación de ejemplos gráficos de cada uno de dichos polígonos. Además, la tarea permite que el estudiante identifique posibles clasificaciones inclusivas entre los polígonos expuestos.

La producción de Edna es representativa en la asignación de varias categorías, a saber: categoría 2.6 [Usar la definición de diversos polígonos para determinar una clasificación de los mismos], categoría 2.10 [Clasificar diferentes polígonos usando propiedades matemáticas], categoría 2.11 [Realizar clasificaciones inclusivas]. En la 2.6, realiza un correcto uso de la definición para elegir la representación gráfica correspondiente; en la 2.10, realiza eficazmente la clasificación usando las propiedades matemáticas de las figuras (esta categoría se 
diferencia de la 1.10, porque la clasificación se hace únicamente mediante atributos físicos-visuales), en razón a que la estudiante toma medidas de lados y ángulos para confirmar lo que está observando.

Figura 2

Producción de Edna en la tarea $n^{\circ} 8$ de la actividad

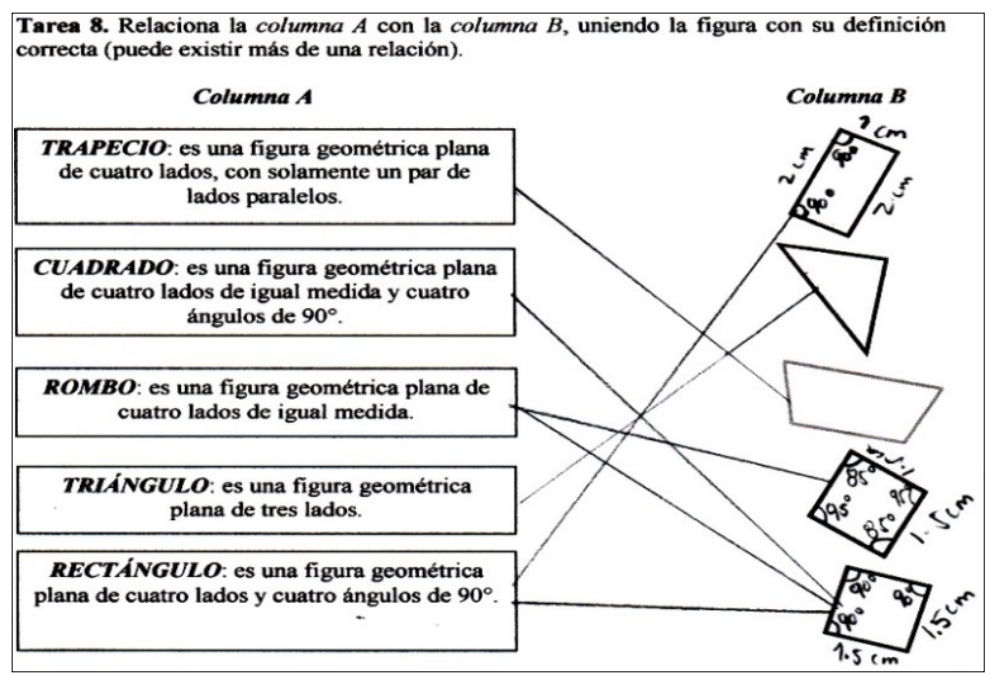

Fuente: elaboración propia

\subsubsection{En la Actividad N²}

El diseño de la tarea $\mathrm{N}^{\circ} 4(\mathrm{a}, \mathrm{b}$ y $\mathrm{c})$ tiene como finalidad realizar clasificaciones inclusivas entre distintas clases de triángulos, según la medida de sus ángulos.

El desarrollo que realiza Zamir en esta tarea muestra su avance en los razonamientos de este tipo. No solamente dibuja los posibles triángulos objeto de la tarea, sino que también justifica los dibujos presentados. Zamir muestra en su producción que puede realizar clasificaciones entre diferentes clases de triángulos; es por esto que se le ha asignado la categoría 2.8 [Usar la definición de triángulos equiláteros, isósceles y escalenos para determinar una clasificación de los mismos]. La categoría 2.8 se diferencia de la categoría 1.10 por que la 2.8 tiene en cuenta la definición (que incluye características matemáticas) y, la 1.10, únicamente atributos físicos.

Figura 3

Producción de Zamir en la tarea $n^{\circ} 4(a, b$ y c) de la actividad 2

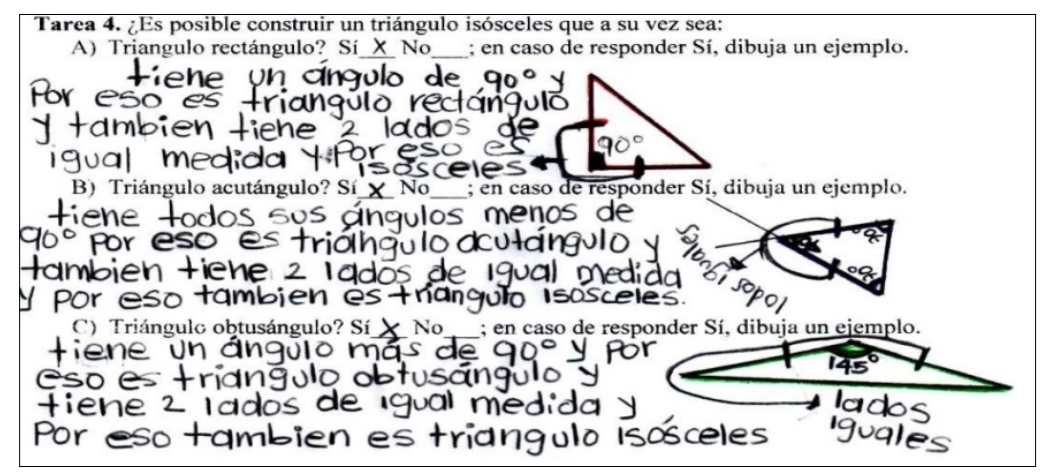

Fuente: elaboración propia

La tarea $\mathrm{N}^{\circ} 10$ se diseñó con el fin que los estudiantes justifiquen si la propiedad de los triángulos, sobre la suma de 180 grados en sus ángulos interiores, es verdad o falsedad. $\mathrm{Y}$, la tarea $\mathrm{N}^{\circ} 11$ que se diseñó para aplicar la propiedad que acaban de conocer. 
La producción de Brayan, en la tarea º 10(a), muestra una forma interesante de "demostración", que realmente es una mostración; además de la producción en la tarea $\mathrm{N}^{\circ} 11$ sobre la aplicación de la propiedad en mención. Al razonamiento en la tarea $\mathrm{N}^{\circ} 10$ se ha asignado categoría 2.12 [Deducir y demostrar propiedades matemáticas de polígonos únicamente realizando verificación mediante ejemplos] y, al razonamiento en la tarea $\mathrm{N}^{\circ} 11$, se ha asignado la categoría $\mathbf{2 . 2}$ [Reconocer aspectos matemáticos específicos de los polígonos, tales como la amplitud de sus ángulos o la medida de sus lados, el paralelismo y la perpendicularidad entre sus lados].

Figura 4

Producción de Brayan en la tarea $\mathrm{n}^{\circ} 10$ (a) y 11 de la actividad 2

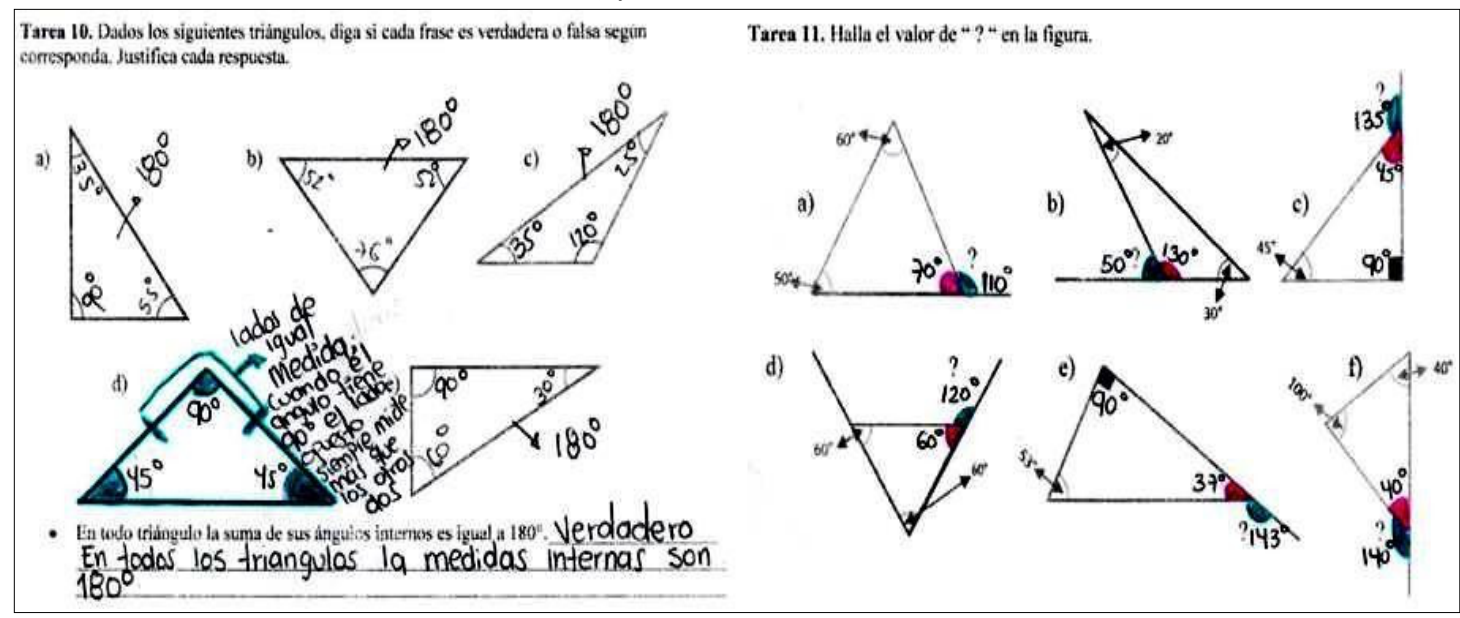

Fuente: elaboración propia

Las producciones de Diana (tarea $N^{\circ} 13$ ) y de Ximena (tarea $N^{\circ} 14$ ) muestran una acertada respuesta en la relación que se puede establecer entre las clasificaciones de triángulos. Se evidencia la estrecha relación que existe entre las clasificaciones de los triángulos que son dominadas por Diana y Ximena. Los razonamientos de las estudiantes recibieron la categoría 2.4 [Comparar y diferenciar polígonos concretamente mediante sus características matemáticas], la categoría 2.8 [Usar la definición de triángulos equiláteros, isósceles y escalenos para determinar una clasificación de los mismos], y la categoría 2.9 [Usar la definición de triángulos acutángulos, obtusángulos y rectángulos para determinar una clasificación de los mismos].

Figura 5

Producción de Diana y Ximena en la

tarea $\mathrm{n}^{\circ} 13$ y 14 de la actividad 2

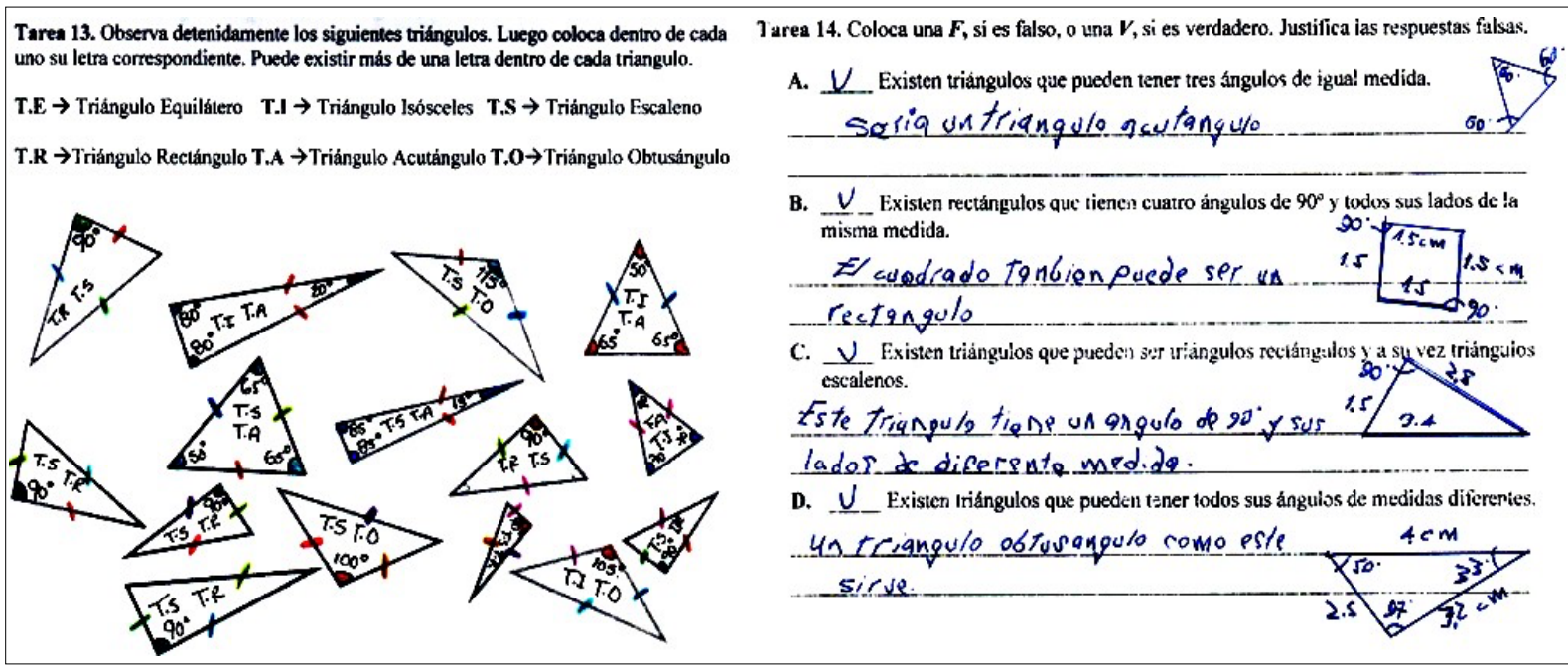


En conclusión, al analizar las producciones de todos los estudiantes participantes en la intervención, se puede destacar que lograron el objetivo propuesto; es decir fortalecieron y adquirieron el concepto de triángulo y las diferentes clasificaciones según la medida de sus lados y según la medida de sus ángulos. En cuanto a los niveles de razonamiento de Van Hiele, se aprecia que los estudiantes han migrado completamente al nivel 2, y usan el nivel 1 de razonamiento sólo como un apoyo a los razonamientos usados en la resolución de las tareas; esto se corresponde con lo planteado por Van Hiele (1999), donde sugiere que, en la medida que el diseño de las actividades para desarrollar un objeto geométrico sean adecuadas, los estudiantes logran esta migración.

\subsubsection{En la Actividad $\mathrm{N}^{\circ} 3$}

El diseño de la actividad $N^{\circ} 5$ incluye como objetivo la selección (clasificación) de cuadriláteros partiendo de la definición de los mismos. El diseño de la tarea incluye la posibilidad que se realicen clasificaciones inclusivas si los participantes las identifican.

La producción de José muestra una clasificación general de los cuadriláteros y una clasificación inclusiva acertada (por ejemplo, incluye al cuadrado como rombo, incluye al trapecio rectángulo como trapecio escaleno). Al razonamiento usado por José se le han asignado la categoría $\mathbf{2 . 6}$ [Usar la definición de diversos polígonos para determinar una clasificación de los mismos] y la categoría 2.11 [Realizar clasificaciones inclusivas].

Figura 61

Producción de José en la tarea $\mathrm{n}^{\circ} 5$ de la actividad 3.

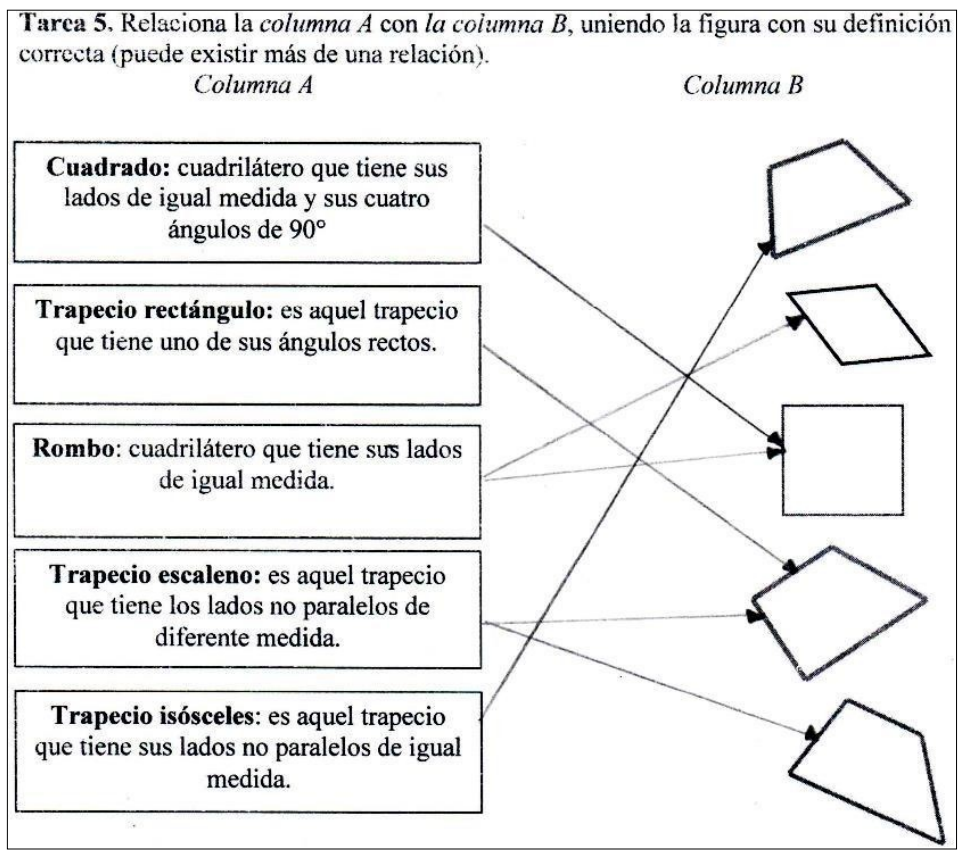

Fuente: elaboración propia

La tarea $\mathrm{N}^{\circ} 9$ propone la deducción de un par de propiedades matemáticas de los cuadriláteros; por un lado, que "las diagonales en el cuadrado y en el rectángulo se interceptan en sus puntos medios" y, por otro, que "las diagonales en el cuadrado y el rombo se interceptan en sus puntos medios de forma perpendicular".

Las producciones de Zamir (primera parte literal a) y Paula (segunda parte literal a y literal b)muestran los razonamientos matemáticos tendientes a plantear las propiedades respecto a relaciones entre las diagonales de los cuadriláteros. De manera similar, Paula (literal b) muestra una aplicación de las propiedades concretamente 
en las tres clases de trapecios. A las producciones de Zamir y Paula se les asignó la categoría 2.12 [Deducir y demostrar propiedades matemáticas de polígonos únicamente realizando verificación mediante ejemplos].

Figura 7

Producción de Xamir en la tarea

$n^{\circ} 9$ y 9 (a) de la actividad 3

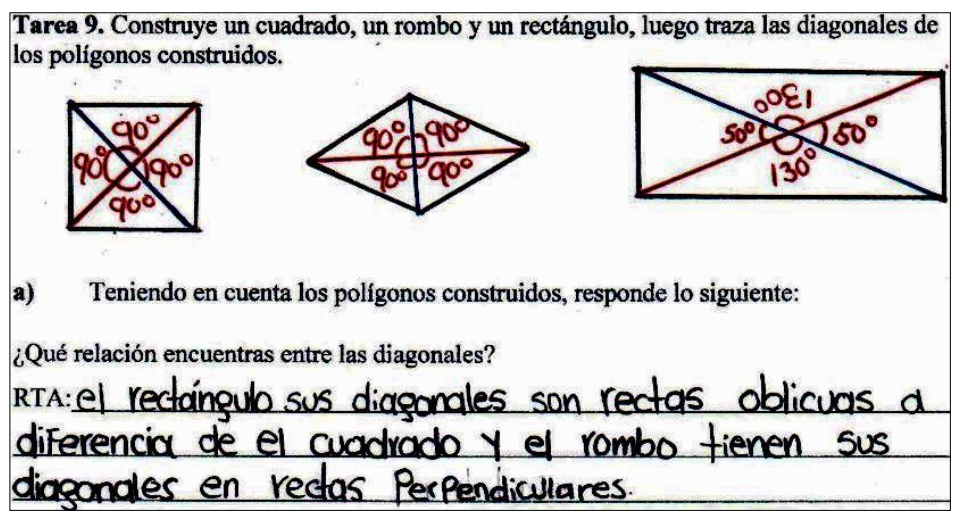

Fuente: elaboración propia

Figura 8

Producción de Paula en la tarea

$n^{\circ} 9$ (a) y 9 (b) de la actividad 3

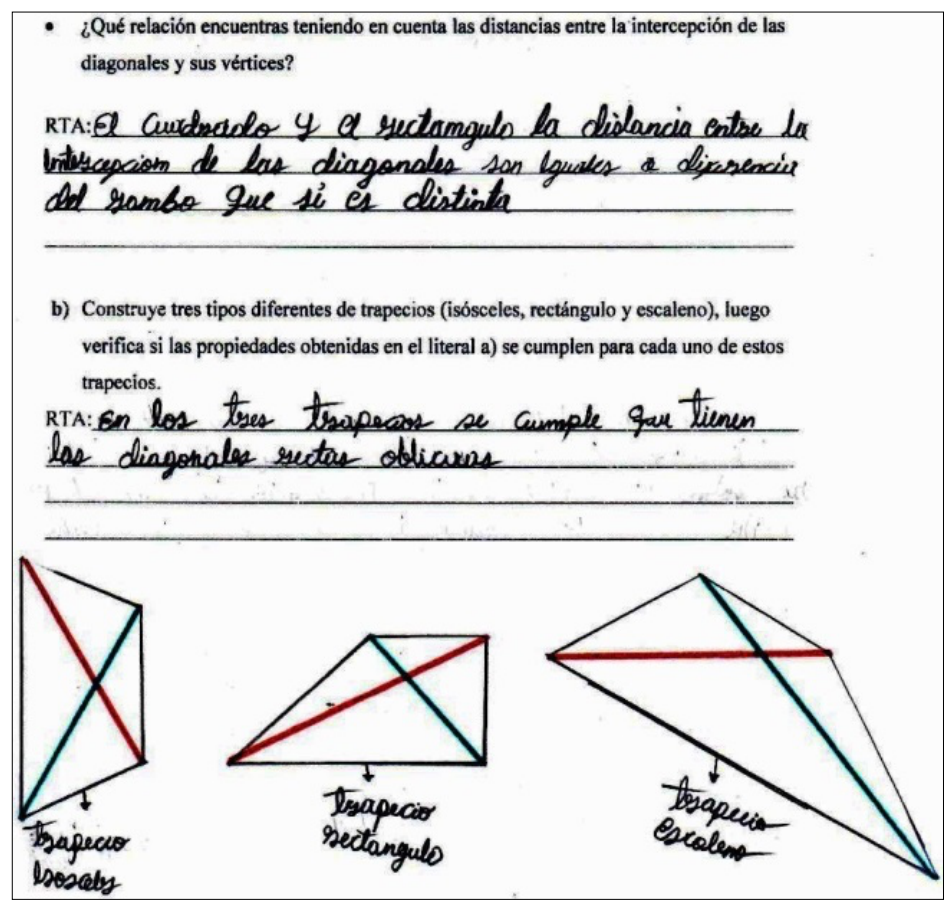

Fuente: elaboración propia

El diseño de tarea $\mathrm{N}^{\circ} 12$ contempla dos objetivos concretos, uno que se reconozcan aspectos matemáticos de los polígonos presentados y, otro, que se realicen clasificaciones de dichos polígonos. 
El desarrollo presentado por Cristian muestra los avances en los objetivos planteados en la tarea; por ejemplo reconoce elementos como el paralelismo, la concavidad, la convexidad y las clases de ángulos, lo que sugiere la asignación de la categoría $\mathbf{2 . 2}$ [Reconocer aspectos matemáticos específicos de los polígonos, tales como la amplitud de sus ángulos o la medida de sus lados, el paralelismo y la perpendicularidad entre sus lados]. Además, al hacer la elección de los polígonos, está clasificándolos según la concavidad o no concavidad, según el paralelismo de sus lados opuestos; estos razonamientos usados por Cristina sugieren la asignación de la categoría 2.10 [Clasificar diferentes polígonos usando propiedades matemáticas].

Figura 29

Producción de Cristian en la tarea $\mathrm{n}^{\circ} 12$ de la actividad 3

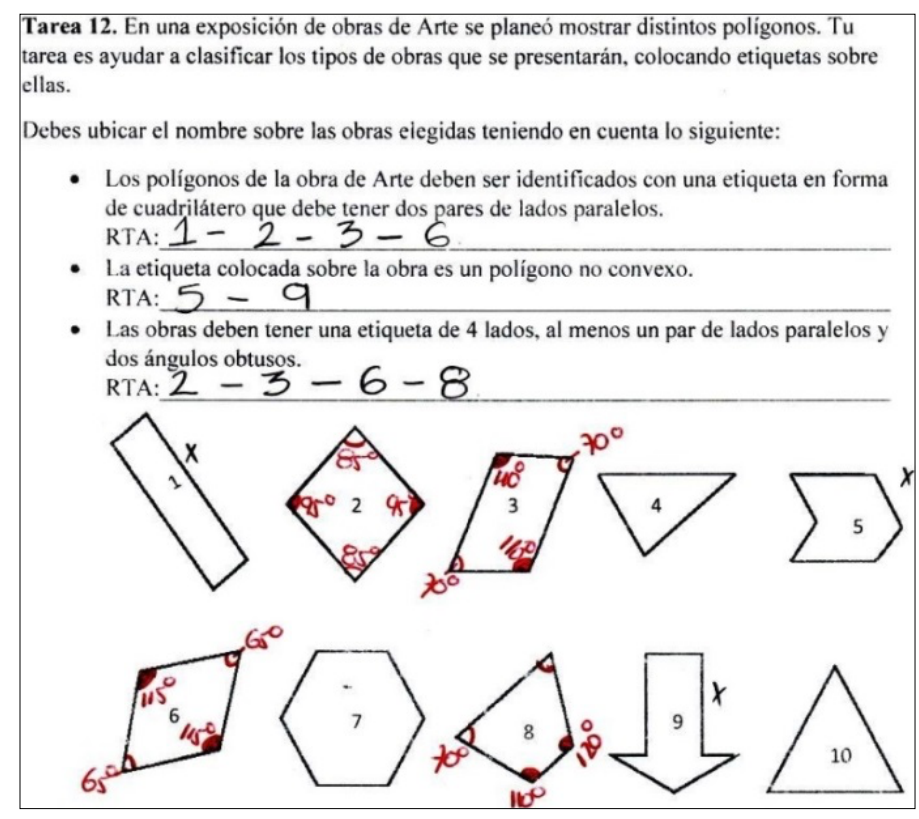

Fuente: elaboración propia

A modo de cierre, analizadas las producciones de todos los estudiantes participantes en la intervención, se puede destacar el logro del objetivo propuesto. Los estudiantes fortalecieron y adquirieron el concepto de cuadrilátero y las diferentes clasificaciones según la concavidad o la conexidad y según el paralelismo de sus lados opuestos. El fortalecimiento de las clasificaciones, en particular, inclusivas se alcanza en la medida que las definiciones de los diferentes objetos geométricos se adquieren adecuadamente (De Villiers, 1994); en particular, cuando las definiciones de los cuadriláteros lo han sido.

Además, las trayectorias de aprendizaje de los estudiantes, en relación a los temas tratados, evolucionaron efectivamente teniendo en cuenta el avance de los estudiantes en los niveles de razonamiento de Van Hiele y en el desarrollo de procesos matemáticos: definición y clasificación.

\section{Conclusiones}

En este capítulo se presentan las principales conclusiones del estudio abordado, fruto de los análisis y resultados realizados.

\subsection{Respuesta a la pregunta de investigación}

Los análisis y resultados realizados en el apartado anterior muestran un avance significativo en las trayectorias de aprendizaje de los estudiantes, particularmente en el desarrollo de los proceso matemáticos de pensamiento: definir (formulación y uso) y clasificar. Esta idea se apoya en las diferentes categorías de análisis que fueron 
surgiendo en las producciones de los estudiantes conforme se avanzaba en el desarrollo de la unidad didáctica experimental. Además, esto se corresponde con la adquisición que alcanzaron los estudiantes de los niveles de razonamiento de Van Hiele 1 y 2; así como también del desarrollo y uso de la definición de los conceptos y las imágenes conceptuales de los mismos.

\subsection{Consecuación de los objetivos planteados}

Los estudiantes comprendieron la relación definición del concepto y la imagen del concepto en los polígonos, los triángulos y los cuadriláteros, no solamente visualmente sino que además matemáticamente; en particular, este resultado concuerda con los hallazgos de Fujita (2012) en especial para el caso de los cuadriláteros. También, se logró evidenciar que los estudiantes comprendieron la importancia y utilidad de formular y usar las definiciones de los conceptos y asumieron las diversas clasificaciones que se presentan en los polígonos, triángulos y cuadriláteros. Especialmente, se pudo confirmar la importancia que tiene la estrategia del uso de ejemplos y no ejemplos de la figura para la formulación de la definición del concepto, lo que sugiere estudios como los de Clements, Sarama, Swaminathan, Weber \& Trawick-Smith (2018) y los de Zaslavsky (2019).

Además, los estudiantes lograron adquirir un lenguaje matemático acorde a las temáticas desarrolladas, lo cual se hizo evidente al momento de intercambiar ideas entre ellos mismos y con el profesor, así como también lograron un mayor control de las imágenes de los conceptos de polígono, triángulo y cuadrilátero superando la influencia limitada que tenían con las figuras prototípicas; este resultado sugiere la riqueza, los desafíos y las posibilidades de razonar con y através de las imágenes de los conceptos.

En general, en los conceptos desarrollados, los estudiantes exhibieron un total y rápido logro de nivel 1 de Van Hiele, como se puede evidenciar en la poca asignación de categorías de análisis en este nivel. Por otra parte, los estudiantes en su totalidad alcanzaron el nivel 2 de Van Hiele, aunque de una manera más lenta si se compara con el logro del nivel 1. Los resultados de este estudio confirman la complementariedad de la imagen y la definición de los conceptos geométricos para el desarrollo de procesos matemáticos de pensamiento.

\subsection{Aportes a la didáctica de las matemáticas}

Uno de los aspectos más importantes a resaltar como resultado de esta investigación, y que con seguridad, es uno de los principales aportes a esta disciplina, es la unidad didáctica diseñada. Los factores estructurantes de la misma, como lo son los modelos de Van Hiele y de Vinner \& Hershkowitz (la definición del concepto versus imagen del concepto), y los procesos matemáticos de pensamiento (definición y clasificación) se constituyen en un ejemplo digno de ser replicado en unidades didácticas de otros objetos matemáticos.

Otro aporte, no menos importante, es el amplio listado de categorías de análisis que se planteó y se logró consolidar con las ejecuciones de los estudiantes en cada una de las tareas de las actividades de la unidad didáctica.

\section{Agradecimientos}

Los autores agradecen a la Vicerrectoría de Investigaciones de la Universidad de Pamplona (Colombia) por el apoyo financiero para el desarrollo del estudio y a la Coordinación de Posgrados de la Facultad de Ciencias Sociales, humanidades y Artes de la Universidad Autónoma de Bucaramanga (Colombia) por el apoyo logístico. También, agradecemos a los miembros del Grupo de Investigación EDUMATEST de la Universidad de Pamplona por los comentarios que hicieron a las versiones previas de este documento. 


\section{Referencias bibliográficas}

Arias, F. (2006). El proyecto de investigación: introducción a la metodología científica. Caracas, Venezuela: Episteme.

Ávila, Ö, (2020). Del «acervo-argumentativo» en el aula: conteos e ideario aritmético (trabajo de investigación doctoral). Universidad de Los Andes, Mérida, Venezuela.

Burger, W.F. \& Shaughnessy, J.M. (1986). Characterizing the Van Hiele levels of development in geometry. Journal for Research in Mathematics Education, 17(1): 31-48. [Traducción por Mạ Luisa Luna (E.U. de Magisterio. Universidad de Cádiz); revisada por Ángel Gutiérrez (Departamento de Didáctica de la Matemática. Universidad de Valencia)].

Cabanne, N. \& Ribaya, M. (2009). Didáctica de la Matemática en el nivel inicial. Buenos Aires, Argentina: Bonum.

Clements, D. H. (2003). Teaching and learning geometry. In J. Kilpatrick,W. G. Martin, \& D. Schifter (Eds.), A research companion to principles and standards for School mathematics (pp. 15-78). Reston, VA: NCTM.

Clements, D. H., Sarama, J., Swaminathan, S., Weber, D., \& Trawick-Smith, J. (2018). Teaching and learning geometry: Early foundations. Quadrante, 27(2), 7-31.

De Villiers, M.D. (1987). Research evidence on hierarchical thinking, teaching strategies and the Van Hiele theory: Some critical comments. Stellenbosch, Sudáfrica: Rumeus.

Fujita, T. (2012). Learners' level of understanding of the inclusion relations of quadrilaterals and prototype phenomenon. The Journal of Mathematical Behavior, 31, 60-72.

Gamboa, R. \& Ballestero, E. (2010). La enseñanza y aprendizaje de la geometría en secundaria: la perspectiva de los estudiantes. Revista Electrónica Educare, 14(2): 125-142.

Goldenberg, E. P., Cuoco, A. A., \& Mark, J. (1998). A role for geometry in genera education. In R. Lehrer, \& D. Chanzan (Eds.), Designing learning environments for developing understanding of geometry and space (pp. 2-44). Mahwah, NJ: Lawrence Erlbaum Associates.

Gonzales, P., Calsyn, C., Jocely, M. K., Kastberg, D., Arafeh, T., Williams, T., et al. (2000). Comparisons of international eighth-grade mathematics and science achievement from a U.S. perspective, 1995 and 1999. NCES 2001-028. Washington, DC: US Department of Education.

Gualdrón, É. (2011). Análisis y caracterización de la enseñanza y aprendizaje de la semejanza figuras planas (tesis doctoral). Universidad de Valencia, Valencia, España.

Gutiérrez, A. \& Jaime, A. (1998). On the assessment of the Van Hiele levels of reasoning. Focus on Learning Problems in Mathematics, 20(2-3): 27-46.

Hershkowitz, R. (1990). Psycological aspects of learning geometry. In P. Nesher, \& J. Kilpatrick (Eds.). Mathematics and cognition. NY, USA: Cambridge University Press.

Hock, T. T., Tarmizi, R. A., Yunus, A. S., \& Ayub, A. F. (2015). Understanding the primary school students' van Hiele levels of geometry thinking in learning shapes and spaces: A Q-methodology. Eurasia Journal of Mathematics, Science and Technology Education, 11(4), 793-802.

Hoffer, A. (1981). Geometry is more than proof. The Mathematics Teacher, 74(1): 11-18.

Hurtado, J. (2000). Metodología de la investigación holística. Caracas, Venezuela: Fundación Sypal.

ICFES. (2011). Informe técnico Saber $5^{\circ}$ y $9^{\circ}$ 2009. Instituto Colombiano para la Evaluación de la Educación. Bogotá, Colombia: Icfes. 
ICFES. (2017a). GUÍAS: Descripción de los niveles de desempeño. Recuperado de: portal.icfes.s3.amazonaws.com/datos/guiasCognitivo/Definicion\%20Niveles\%20de\%20Desempe\%C3\%B1o .pdf

ICFES. (2017b). Publicación de resultados Saber $3^{\circ}, 5^{\circ}$ y $9^{\circ}$. I.E.T.A. VICENTE HONDARZA - Morales: histórico. Recuperado de:

http://www2.icfesinteractivo.gov.co/ReportesSaber359/historico/reporteHistoricoComparativo.jspx

Jaime, A. (1993). Aportaciones a la interpretación y aplicación del modelo de Van Hiele: la enseñanza de las isometrías del plano. La evaluación del nivel de razonamiento (tesis doctoral). Universidad de Valencia, Valencia, España.

Quintero, M. (2020). Fortalecimiento de los procesos matemáticos de pensamiento: definición y clasificación en estudiantes de $5^{\circ}$ grado de la Institución Educativa Técnica Agropecuaria Vicente Hondarza de Morales (Bolívar) (tesis de matestría). Universidad Autónoma de Bucaramanga, Bucaramanga, Colombia.

Marín, J. D. (2018). Investigar en educación y pedagogía: sus fundamentos epistemológicos y metodológicos. Bogotá, Colombia: Editorial Magisterio.

NCTM. (2000). Principles and standards for school mathematics. Reston, VA: National Council of Teachers of Mathematics.

Pegg, J. \& Davey, G. (1998). Interpreting student understanding in geometry: A synthesis of two models. En R. Lehrer y D. Chazan (Eds.), Designing Learning Environments for Developing Understanding of Geometry and Space (pp. 109-135). Mahwah, NJ: Lawrence Erlbaum Associates.

Porlán, R. \& Martin, J. (1991). El Diario del Profesor. Sevilla, España: Diada. Puig, L. (1996). Elementos de resolución de problemas. Granada, España: Comares.

Russell, D. (2018, September 4). What is geometry? Recuperado de https://www.thoughtco.com/what-isgeometry-2312332.

Van Hiele, P.M. (1957). El problema de la comprensión en conexión con la comprensión de los escolares en el aprendizaje de la geometría (tesis doctoral). Utrecht, Holanda: Universidad de Utrecht. Recuperado de: http://www.uv.es/angel.gutierrez/aprengeom/archivos2/VanHiele57.pdf

Van Hiele, P.M. (1986). Structure and insight. A theory of mathematics education. NY, USA: Academic Press.

Van Hiele, P. M. (1999). Developing geometric thinking through activities that begin with play. Teaching Children Mathematics, 5(6): 310-316.

Vinner, S. (1983). Concept definition, concept image and the notion of function. International Journal of Mathematical Education in Science and Technology, 14: 293-305.

Vinner, S. (1991). The role of definitions in the teaching and learning of mathematics. In D. Tall. (Ed.), Advanced mathematical thinking (pp. 65-81). Dordrecht, Netherlands: Kluwer Academic Publishers.

Vinner, S. \& Hershkowitz, R. (1983). On concept formation in geometry, Zentralblatt für Didaktik der Mathematik, 83 (1): 20-25.

Wu, D. B., \& Ma, H. L. (2006). The distributions of van Hiele levels of geometric thinking among 1st through 6th graders. In Proceedings 30th conference of the international group for the psychology of mathematics education (Vol. 5, pp. 409-416).

Zaslavsky, O. (2019). There more to examples than meets the eye: thinking with and through mathematical examples in different settings. Journal of Mathematical Behavior, 53: 245-255.

Esta obra está bajo una Licencia Creative Commons

Attribución-NoCommercial 4.0 International 